\title{
Volumetría de fascículos cerebrales en población sana
}

\section{Brain fascicles volumetry in healthy population}

José Fernando Hernández ${ }^{1}$ Leonardo Bello-Dávila ${ }^{1}$ Sergio Francisco Ramírez ${ }^{1} \quad$ Jorge Marin ${ }^{1}$ Jorge Rudas $^{2}$ Edgar G. Ordóñez-Rubiano ${ }^{1}$ Jenny Vicuña Vanegas ${ }^{1}$

${ }^{1}$ Hospital Universitario Infantil de San José, Bogotá, Colombia
${ }^{2}$ Departamento de Ciencias, Universidad Nacional de Colombia,
Bogotá, Colombia

Rev Argent Radiol 2019;83:95-101.

\author{
Address for correspondence Leonardo Bello-Dávila, MD, Hospital \\ Universitario Infantil de San José, Bogotá, Colombia \\ (e-mail: mai-8070@hotmail.com).
}

\begin{abstract}
Resumen
Palabras Clave

- anisotropía

- cognición

- imagen con tensor de diffusion

- sustancia blanca

- tractografía

Objetivo Desde la aparición de la tractografía, al tratarse de una técnica no invasiva y que usa la ampliamente conocida resonancia magnética (RM), el estudio de la sustancia blanca se ha facilitado. A partir de eso, se han logrado grandes descubrimientos en cuanto a fascículos cerebrales involucrados en funciones cognitivas. Sin embargo, su evaluación sigue siendo subjetiva y depende de la experiencia y entrenamiento del evaluador, razón por la cual se ha limitado su aplicabilidad en la práctica clínica. En ese sentido, es conveniente parametrizar volúmenes cerebrales de sustancia blanca en población sana a través de una herramienta electrónica que se pueda reproducir y así poderlo aplicar en enfermos.

Materiales y métodos Se reportan 10 sujetos sanos desde el punto de vista neurológico. Para cada sujeto, se adquirieron imágenes ponderadas por difusión y los resultados se visualizaron mediante FiberNavigator (http://scilus.github.io/ fibernavigator). Finalmente, esa misma herramienta fue utilizada para purificar los fascículos objeto de estudio y realizar el conteo de las fibras.

Resultados Se obtuvieron valores de volumetría del fascículo longitudinal superior (FLS), fascículo longitudinal inferior (FLI), fascículo frontoccipital inferior (FFI), fascículo uncinado (FU) y fascículo del cíngulo (FC), identificando que no existen diferencias estadísticamente significativas entre el número de fibras que componen los fascículos cerebrales.

Discusión Los resultados alcanzados de la anatomía y la direccionalidad de fibras de los fascículos cerebrales de este estudio coinciden con el resto de la evidencia publicada hasta el momento, sin encontrar diferencias en cuanto a su organización y recorrido. Conclusión Aunque esos resultados no sirven como valores de referencia para ser aplicados en pacientes con patología neurológica, brindamos información inexistente hasta el momento, con ese equipo en específico y la reproducción entre los distintos usuarios y el software.
\end{abstract}

received

April 3, 2018

accepted

April 11, 2019
DOI https://doi.org/

10.1055/s-0039-1693672.

ISSN 1852-9992.
Copyright $\odot$ 2019, Sociedad Argentina de Radiología. Publicado por Thieme Revinter Publicações Ltda., Rio de Janeiro, Brazil. Todos los derechos reservados.
License terms

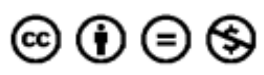




\begin{abstract}
Keywords

- anisotropy

- cognition

- diffusion tensor imaging

- diffusion tractography

- white matter

Objective Since the appearance of tractography, as it is a non-invasive technique and uses the widely known magnetic resonance (MR), the study of white matter has been facilitated. After this, great discoveries have been made regarding the brain fascicles involved in cognitive functions. However, its evaluation continues to be subjective and depends on the evaluator's experience and training. That the reason why its applicability has been limited in clinical practice. Because of this, it is convenient to parametrize cerebral volumes of white matter in healthy population through an electronic, reproducible tool that could be applied in patients.

Materials and Methods Ten neurologically healthy subjects are reported, for each subject we acquired images weighted by diffusion and the results were visualized by means of FiberNavigator (http://scilus.github.io/fibernavigator). Finally, this same tool was used to purify the fascicles under study and perform the fiber count.

Results Volumetric values of the upper longitudinal fasciculus, inferior longitudinal fasciculus, inferior frontoccipital fasciculus, uncinated fasciculus and cingulate fasciculus were obtained, identifying that there are not statistically significant differences in the number of fibers that make up the cerebral fascicles.

Discussion The results achieved of the anatomical and fiber directionality of the cerebral fascicles of this study, coincide with the rest of the evidence published up to now, without finding differences regarding its organization and route.

Conclusion Although these results do not serve as reference values to be applied in patients with neurological pathology, do we provide nonexistent information so far, with this specific equipment and the reproducibility between the different users and the software.
\end{abstract}

\section{Introducción}

Investigaciones recientes han descrito diferentes tractos que asocian múltiples regiones corticales, siendo esa la base de la cognición humana. ${ }^{1}$ Esto ha llevado a los investigadores a dirigir sus estudios hacia el análisis de los fenómenos subyacentes de la sustancia blanca, teniendo en cuenta que no se conocen con exactitud las vías de conexión subcortical. Gracias a los avances en resonancia magnética $(R M)$, se ha logrado conocer aún mejor la estructura y función del cerebro, permitiendo aislar tractos implicados en procesos cognitivos. ${ }^{2}$ Desde el inicio de las imágenes de tensor de difusión (DTI, por su sigla en inglés), no se ha discutido su utilidad en neurorradiología, ya que la técnica representa la suma vectorial de las moléculas de agua, dando una direccionalidad gráfica de los axones, representada en colores como en el mapa de anisotropía. ${ }^{3}$ La tractografía corresponde entonces a la representación en 3D del DTI, lo que permite visualizar la trayectoria cerebral subcortical y se ha convertido en un elemento de apoyo diagnóstico fundamental para la neurología. ${ }^{4}$ A partir de esas evaluaciones, se logró identificar que la integridad de la sustancia blanca tiene relación con la preservación de las actividades cognitivas. $^{5}$

La tractografía es un estudio no invasivo y permite evaluar la integridad de tractos largos. De esa manera, empezó a ser útil en neurocirugía para la planeación prequirúrgica y para reducir secuelas postquirúrgicas, siendo los gliomas y la vía corticoespinal los más estudiados. $^{6,7}$ Publicaciones con DTI hacen referencia a patologías neurológicas que presentan algún tipo de alteración en los tractos de sustancia blanca. Tal es el caso de la epilepsia del lóbulo temporal, donde se ha observado una reducción de la anisotropía fraccional (AF) del fascículo longitudinal superior (FLS) y en la enfermedad de Alzheimer, el fascículo uncinado (FU) derecho presenta disminución en la AF con respecto al contra lateral. , $^{8,9}$

A pesar de eso, la valoración de la tractografía suele ser subjetiva y dependiente del entrenamiento del personal clínico implicado. Además, no disponemos de parámetros de normalidad en muchos casos (volúmenes de paquetes de tractos, longitudes, etc.) requeridos para establecer puntos de comparación en estudios poblacionales. De acuerdo con lo anterior, existe la necesidad de parametrizar valores en población sana, razón por la cual presentamos la volumetría de los fascículos cerebrales más importantes implicados en funciones cognitivas en sujetos sanos.

\section{Materiales y métodos}

Se reportaron 10 sujetos de entre 18 y 51 años de edad (siete hombres y tres mujeres), sanos desde el punto de vista neurológico (evaluación cognitiva de Montreal (MoCA) normal) y sin ningún tipo de sintomatología o antecedente patológico, a los que se les realizó RM cerebral con tractografía reportada como normal, leída por un neurorradiólogo entrenado en esa técnica. 


\section{Adquisición de imágenes}

Se realizó un estudio completo de RM en equipo 1.5 Teslas (Signa Excite HDXT, GE Healthcare, Milwaukee, WI, EEUU), con una bobina de cabeza. De cada paciente se capturaron las siguientes secuencias: 1) Secuencia estructural ponderada en $\mathrm{T} 1$; 2) Secuencia ponderada en T2 axial; 3) Secuencia ponderada en difusión (DWI, por su sigla en inglés). Cada imagen estructural ponderada en T1 tiene 140 cortes ( $1 \mathrm{~mm}$ de grosor, sin GAP (espacio libre), matrix $=320 \times 192, \mathrm{TR}=$ $650 \mathrm{~ms}, \mathrm{TE}=22 \mathrm{~ms}, \mathrm{FOV}=22)$ y tiempo de adquisición $=$ 2 min y 35 segundos, cubriendo todo el volumen cerebral. Las imágenes estructurales ponderadas en T2 contienen 22 cortes (6 mm de grosor, GAP $1 \mathrm{~mm}$ (espacio libre), matrix $=320 \times 256, \mathrm{TR}=6,000 \mathrm{~ms}, \mathrm{TE}=97,44 \mathrm{~ms}, \mathrm{FOV}=24)$ y tiempo de adquisición $=1$ min y 20 segundos, cubriendo todo el volumen cerebral. Cada imagen de DWI tiene 30 cortes ( $2 \mathrm{~mm}$ grosor, sin espacio libre, matrix $=128 \times 128, \mathrm{TR}=1000$, $\mathrm{TE}=102,3 \mathrm{~ms}$, flip angle $=90)$ con 24 direcciones con técnica echo planarimaging (EPI) y tiempo de adquisición $=6 \mathrm{~min}$, cubriendo todo el volumen cerebral.

\section{Preprocesado}

Inicialmente, las DWI fueron ajustadas con el fin de suprimir artefactos derivados de la adquisición y estandarizar las medidas. Para ello, se aplicó la siguiente secuencia de pasos: alineamiento manual entre DWI y T1, reslice de las DWI para obtener voxeles de tamaño isométrico, co-registro entre imagen de difusión e imagen estructural, corrección de corrientes de Eddy (https://fsl.fmrib.ox.ac.uk/fsl/fslwiki/ eddy), extracción de cerebro y filtrado a través del algoritmo de non-local-mean (http://nipy.org/dipy/examples_built/ denoise_nlmeans.html).

\section{Postprocesado}

Luego del preprocesamiento y con el fin de computar la tractografía derivada de las DWI, se estimó el modelo de tensor por difusión, se generaron los mapas de AF y difusividad media y se generó la tractografía a través de un algoritmo de tracking determinístico basado en EuDX en un campo tensorial.

\section{Visualización}

Se utilizó FiberNavigator para las visualizaciones y la parcelación manual de cada tracto. ${ }^{10}$ Para la ubicación de las regiones de interés (ROI, por su sigla en inglés), nos basamos en el atlas de Wakana y col. ${ }^{11}$ El conteo de fibras se realizó con la herramienta elipsoide y cuboide de FiberNavigator. Así se garantiza el conteo del mayor número de fibras de sustancia blanca que compone el fascículo.

El promedio volumétrico del fascículo y del valor AF se calculó mediante la media aritmética, que es la suma de todos los valores del tracto en cada hemisferio, dividido por el número de sujetos. El análisis estadístico se hizo mediante un test no paramétrico para dos muestras independientes. Particularmente, se utilizó un test U de Mann-Whitney, con un nivel de confianza de al menos el 5\%. Ese test no contempla, a priori, ningún supuesto respecto a la distribución o la naturaleza de los datos. Este trabajo de investigación sigue los lineamientos internacionales relacionados con las recomendaciones para investigación con seres humanos consignados en la declaración de Helsinki (última revisión, Brasil 2013) y el Informe Belmont. Por tal razón, todos los pacientes firmaron el consentimiento informado, aprobado por el comité de ética de la Fundación Universitaria de Ciencias de la Salud.

\section{Resultados}

Se logró aislar 100 tractos de sustancia blanca, en 10 sujetos sanos, mediante imágenes DWI, se obtuvo la AF y un valor volumétrico de los siguientes fascículos cerebrales en cada hemisferio; fascículo longitudinal superior (FLS), fascículo longitudinal inferior (FLI), fascículo frontoccipital inferior (FFOI), fascículo uncinado (FU) y fascículo del cíngulo (FC). En la $\mathrm{AF}$ que se describe en la - Tabla 1, no se encontraron diferencias interhemisféricas de los tractos superiores a 0,03 y en sus valores promedios tampoco se superó esa cifra, razón por la cual no hubo diferencias estadísticamente significativas.

Mediante la tractografía, se consiguió el conteo de cada fibra que conforma el tracto y, de esa manera, se llegó a tener un volumen del fascículo cerebral estudiado, además de un valor promedio descrito en la - Tabla 2. Dichos valores promedio mostraron grandes diferencias volumétricas interhemisféricas de un mismo fascículo (ejemplo: FFOID: 526y FFOII: 575), pero en el análisis estadístico de los resultados, no se encontraron diferencias estadísticamente significativas, como tampoco fue evidente con la AF. En ese punto, hay que resaltar que, a pesar de que algunos fascículos revelaron grandes diferencias en la media aritmética (valor promedio de cada tracto) reportado en la - Tabla 2, ese valor no es adecuado para anotar diferencias volumétricas estadísticamente significativas, debido que esa medida estadística es un valor que está influenciado por los extremos de la distribución.

\section{Fascículo longitudinal superior}

La distribución anatómica de este tracto va en dirección del lóbulo frontal inferior, pasando sus fibras por el parietal, y termina en el temporal superior. En la región frontal anterior confluye junto con el FU y FFOI, haciendo difícil su aislamiento en esa zona, por lo que su siembra se realizó más posterior en su recorrido frontal. Usando el mapa de anisotropía en corte coronal como referencia para determinar la direccionalidad de las fibras, se realizaron las siembras del FLS en dos ROI. En la - Figura 1, se muestra este fascículo en corte sagital para una mejor apreciación de su recorrido.

\section{Fascículo longitudinal inferior}

Este tracto de asociación posee dos porciones: una superficial y una profunda que conecta el polo anterior del lóbulo temporal con el extremo cortical del lóbulo occipital, donde se une con fibras del FFI. Ese fascículo se logró identificar haciendo siembras en un corte sagital en dos ROI, basados en un mapa de anisotropía que permite ver el recorrido de las fibras de sustancia blanca (-Fig. 2). 
Tabla 1 Fracción de anisotropía de fascículos cerebrales

\begin{tabular}{|l|l|l|l|l|l|l|l|l|l|l|l|}
\hline Sujeto & Genero & FLSD & FLSI & FLID & FLII & FFOID & FFOII & FUD & FUI & FCGD & FCGI \\
\hline 1 & M & 0,43 & 0,48 & 0,51 & 0,51 & 0,43 & 0,4 & 0,47 & 0,47 & 0,47 & 0,45 \\
\hline 2 & F & 0,59 & 0,62 & 0,51 & 0,49 & 0,48 & 0,45 & 0,45 & 0,46 & 0,41 & 0,38 \\
\hline 3 & M & 0,43 & 0,45 & 0,54 & 0,54 & 0,48 & 0,46 & 0,48 & 0,49 & 0,41 & 0,38 \\
\hline 4 & F & 0,43 & 0,42 & 0,49 & 0,49 & 0,46 & 0,45 & 0,31 & 0,33 & 0,41 & 0,42 \\
\hline 5 & F & 0,41 & 0,42 & 0,52 & 0,52 & 0,45 & 0,43 & 0,51 & 0,52 & 0,46 & 0,42 \\
\hline 6 & M & 0,48 & 0,48 & 0,41 & 0,39 & 0,34 & 0,31 & 0,41 & 0,41 & 0,45 & 0,44 \\
\hline 7 & M & 0,51 & 0,52 & 0,52 & 0,51 & 0,44 & 0,47 & 0,45 & 0,48 & 0,5 & 0,48 \\
\hline 8 & M & 0,48 & 0,45 & 0,52 & 0,52 & 0,44 & 0,42 & 0,52 & 0,54 & 0,42 & 0,44 \\
\hline 9 & M & 0,51 & 0,53 & 0,47 & 0,47 & 0,44 & 0,46 & 0,52 & 0,53 & 0,43 & 0,45 \\
\hline 10 & M & 0,46 & 0,48 & 0,49 & 0,51 & 0,48 & 0,48 & 0,51 & 0,51 & 0,45 & 0,42 \\
\hline
\end{tabular}

Abreviaturas: F, femenino; FCGD, fascículo cíngulo derecho; FCGI, fascículo cíngulo izquierdo; FFOID, fascículo frontoccipital inferior derecho; FFOIl, fascículo frontoccipital inferior izquierdo; FLI, fascículo longitudinal inferior izquierdo; FLID, fascículo longitudinal inferior derecho; FLSD, fascículo longitudinal superior derecho; FLSI, fascículo longitudinal superior izquierdo; FUD, fascículo uncinado derecho; FUI, fascículo uncinado izquierdo; M, masculino.

Tabla 2 Conteo de fibras de fascículos cerebrales

\begin{tabular}{|l|l|l|l|l|l|l|l|l|l|l|l|}
\hline Sujeto & Genero & FLSD & FLSI & FLID & FLII & FFOID & FFOII & FUD & FUI & FCGD & FCGI \\
\hline 1 & M & 597 & 538 & 1144 & 1169 & 520 & 617 & 314 & 216 & 625 & 570 \\
\hline 2 & F & 557 & 568 & 905 & 936 & 512 & 610 & 296 & 244 & 425 & 510 \\
\hline 3 & M & 562 & 575 & 921 & 934 & 472 & 574 & 333 & 293 & 510 & 580 \\
\hline 4 & F & 520 & 533 & 935 & 944 & 580 & 623 & 244 & 350 & 530 & 564 \\
\hline 5 & F & 542 & 567 & 980 & 995 & 543 & 577 & 288 & 296 & 435 & 467 \\
\hline 6 & M & 556 & 570 & 1055 & 1050 & 412 & 464 & 306 & 318 & 607 & 416 \\
\hline 7 & M & 532 & 551 & 907 & 922 & 580 & 645 & 276 & 252 & 623 & 645 \\
\hline 8 & M & 556 & 580 & 1009 & 940 & 474 & 511 & 303 & 230 & 895 & 970 \\
\hline 9 & M & 524 & 518 & 895 & 975 & 592 & 571 & 329 & 353 & 656 & 687 \\
\hline 10 & M & 588 & 560 & 966 & 990 & 579 & 564 & 316 & 322 & 613 & 640 \\
\hline & Promedio & 553,40 & 556 & 971,7 & 985,5 & 526,4 & 575,6 & 300,5 & 287,4 & 591,9 & 604,9 \\
\hline
\end{tabular}

Tabla 3 Diferencias estadísticas por test de Mann-Whitney

\begin{tabular}{|l|}
\hline Conteo de tractos \\
\hline FLSD - FLSI $->p=0.311522765419$ \\
\hline FLID - FLII $->p=0.21367765694890384$ \\
\hline FFOID - FFOII $->p=0.0928556402224$ \\
\hline FUD - FUI $->p=0.338677972903$ \\
\hline FCGD - FCGI $->p=0.469860183474$ \\
\hline Anisotropía Fraccional \\
\hline FLSD - FLSI $->p=0.337225774999$ \\
\hline FLID - FLII $->p=0.423562261019$ \\
\hline FFOID - FFOII $->p=0.310067765958$ \\
\hline FUD - FUI $->p=0.271762914193$ \\
\hline FCGD - FCGI $->p=0.270685104777$ \\
\hline
\end{tabular}

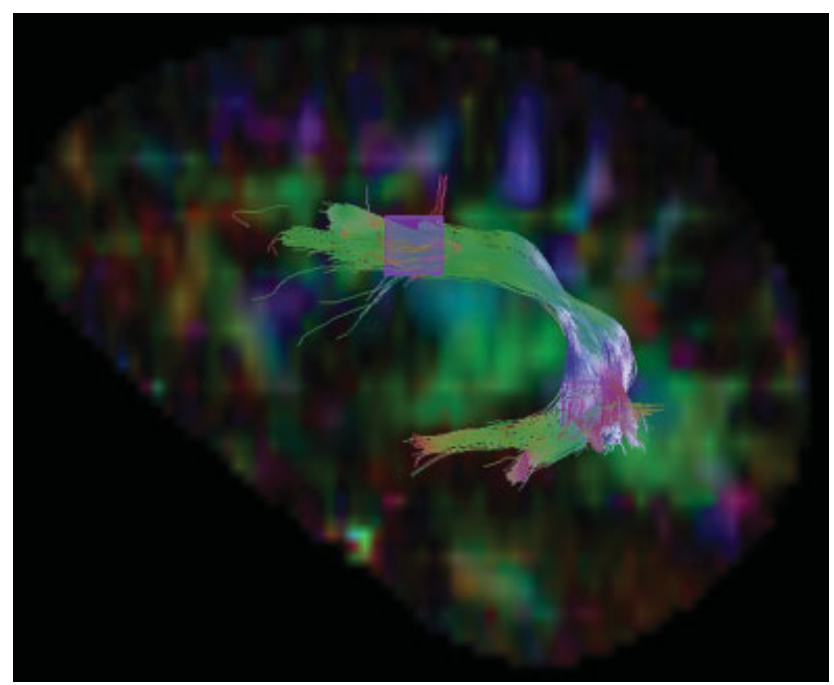

Fig. 1 FLS izquierdo sobre un fondo de mapa de AF en corte sagital. Con la técnica cuboide se hizo la siembra en dos ROI, el primero en la región frontal y el segundo en la región temporal. 


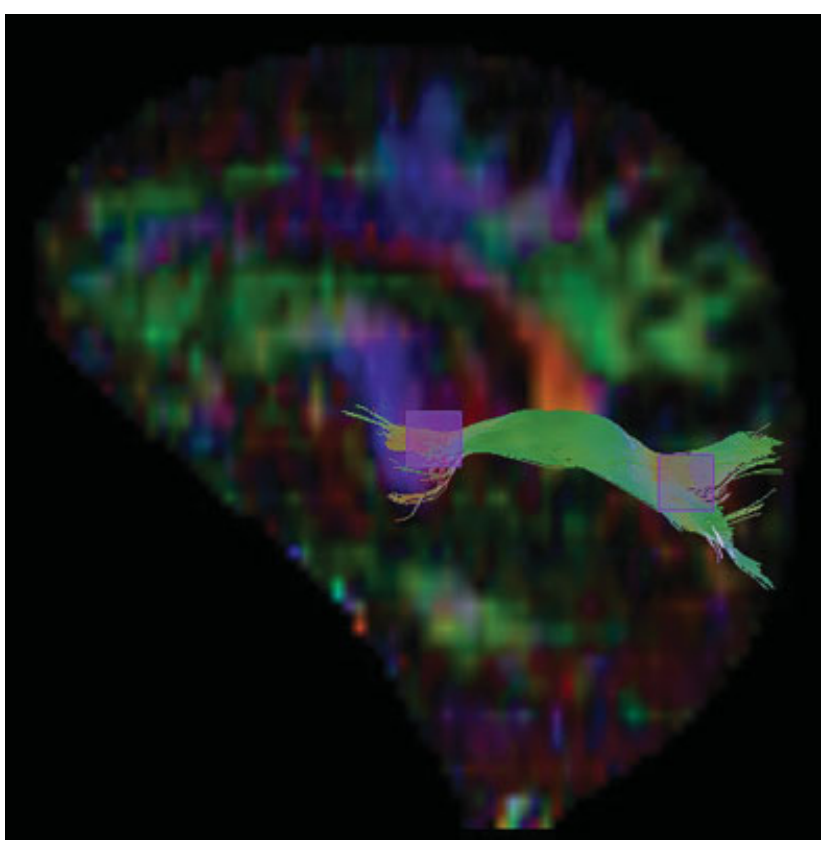

Fig. 2 FLI izquierdo sobre un fondo de mapa de AF en corte sagital. Con la técnica cuboide se hizo la siembra en dos ROI, el primero en la región temporal y el segundo en la región occipital.

\section{Fascículo frontoccipital}

Esas fibras de sustancia blanca conectan el lóbulo occipital con el frontal. Su recorrido inicia en el polo de la corteza occipital, pasando por el lóbulo temporal para finalmente terminar en las áreas orbito frontal y frontopolar. Se aisló ese fascículo basado en el mapa de anisotropía en corte axial mediante la siembra de dos ROI. En la - Figura 3 se puede apreciar completamente su dirección en un corte sagital.

\section{Fascículo uncinado}

Las fibras del FU, denominado así por su forma similar a un gancho y también llamado frontotemporal, asocia de manera

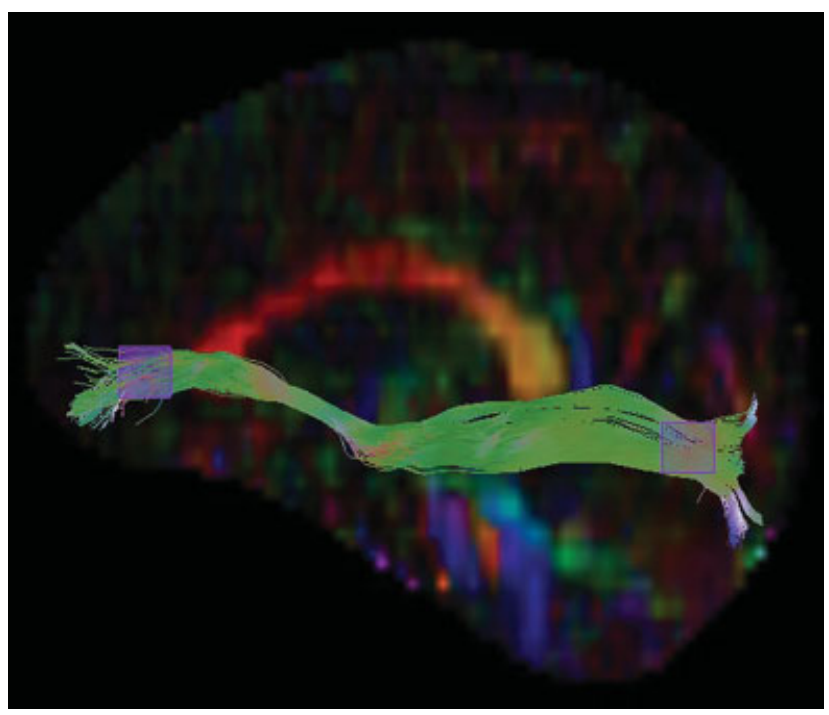

Fig. 3 FFI izquierdo sobre un fondo de mapa de AF en corte sagital. Con la técnica cuboide se hizo la siembra en dos ROI, el primero en la región frontal inferior y el segundo en la región occipital.

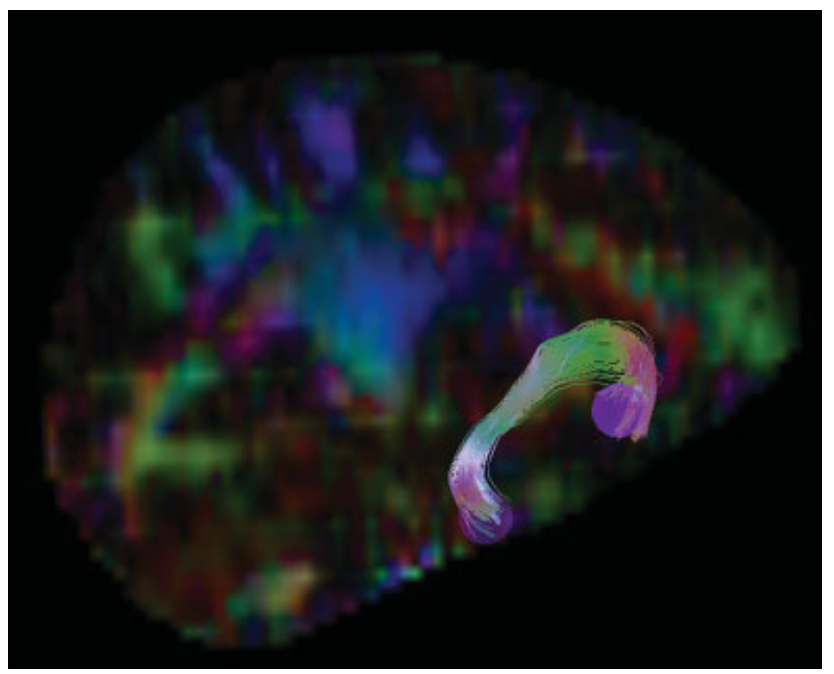

Fig. 4 FU derecho sobre un fondo de mapa de AF en corte sagital. Con la técnica elipsoide se hizo la siembra en dos ROI, el primero en la región frontal inferior y el segundo en la temporal superior.

bidireccional el polo anterior de lóbulo temporal superior con el área orbito frontal. Se ubicaron dos ROI en corte coronal sobre un mapa de anisotropía y su aislamiento está visualizado en la - Figura 4, pero sobre un corte sagital para que de esa manera se pueda observar su forma de gancho.

\section{Fascículo del cíngulo}

Implicado con el sistema límbico, este fascículo posee fibras en Uy de proyección. Las primeras llevan la dirección de todo el giro del cíngulo formando una especie de arco, conectando áreas del prefrontal hasta la zona parahipocampal, alcanzando el uncus del lóbulo temporal. Las fibras en U que inician en el área subcallosa y se conectan con el lóbulo frontal, parietooccipital y temporal. Los dos ROI para ese fascículo se obtuvieron en corte sagital del mapa de anisotropía (-Fig. 5).

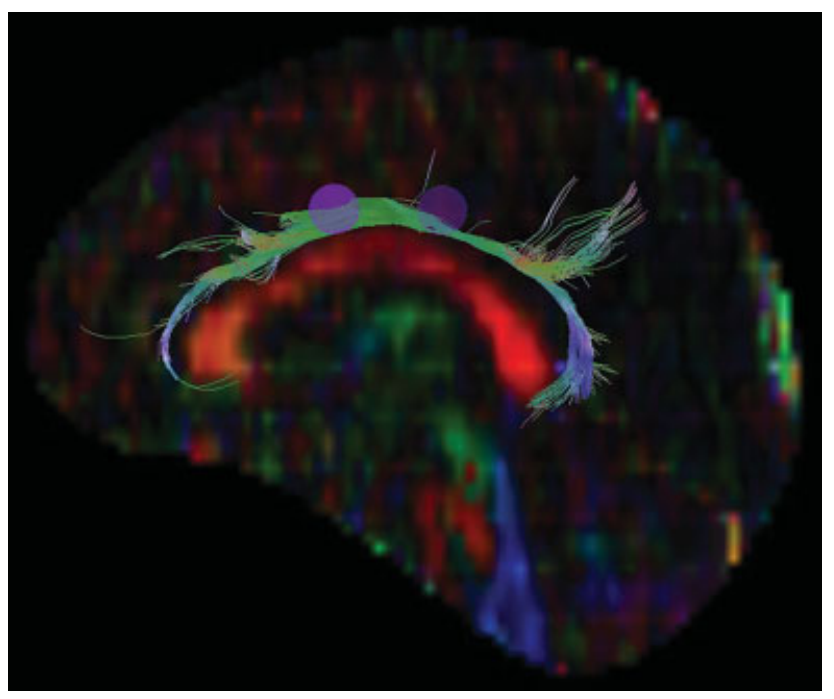

Fig. 5 FC derecho sobre un fondo de mapa de AF en corte sagital. Con la técnica cuboide se hizo la siembra en dos ROI en la región frontal. 


\section{Discusión}

Los resultados alcanzados de la anatomía y la direccionalidad de fibras de los fascículos cerebrales de este estudio coinciden con el resto de la evidencia publicada hasta el momento, sin encontrar diferencias en cuanto a su organización y recorrido. ${ }^{12,13}$ Se escogieron esos cinco fascículos, ya que han sido los más estudiados e involucrados en funciones cognitivas. Destacamos el hecho de poder aislar 100 tractos de manera manual en la población sana, lo que implica el conocimiento cuidadoso de la neuroanatomía radiológica por DTI para que se pueda reproducir el estudio a través de esa técnica, así como el conocimiento óptimo de la herramienta electrónica utilizada. No incluimos en la muestra sujetos mayores de 65 años, teniendo en cuenta que el envejecimiento se ha asociado al deterioro cognitivo por cambios en la sustancia blanca. ${ }^{14,15}$

No se logró identificar alguna dominancia de la sustancia blanca existente entre el hemisferio izquierdo y derecho desde el punto de vista estructural, más allá del funcional en relación con áreas corticales especializadas del lado izquierdo ya conocidas en la literatura, debido a que el resultado de los hallazgos estadísticos no permite hacer esa afirmación. Sin embargo, el estudio de conectoma de lenguaje usó 20 sujetos masculinos sanos, a los que se les cuantificaron ocho fascículos de sustancia blanca, encontrándose una asimetría hacia la izquierda del volumen del fascículo arqueado (FA), FFOI y longitudinal medio, además de extensas conexiones corticales, por lo que no solo estarían implicados exclusivamente en el lenguaje, sino que apoyan otras habilidades cognitivas. ${ }^{16}$ En contraposición a la información anterior, el estudio de Thiebaut y col., ${ }^{17}$ con 40 sujetos sanos, reportó diferencias estadísticamente significativas de asimetría hacia la derecha del FA y el FFOI. Esos resultados contradictorios quizás se den por las diferencias entre los equipos y las técnicas utilizados a la hora de realizar la tractografía, y es por eso que tampoco podemos sacar conclusiones con respecto a las diferencias entre géneros (hombre - mujer), aunque Thiebaut y col. en su estudio confirmó que hay más asimetría hacia la izquierda del FA en hombres. Por otro lado, con la técnica de DTI, se ha determinado un mayor índice de AF en hombres en especial en el FLS y FLI. ${ }^{18}$

Es por eso que hasta el momento no podemos tomar a la tractografía como un método totalmente confiable a la hora de estudiar la sustancia blanca, teniendo en cuenta la heterogeneidad de las patologías, las diferencias entre cada individuo y los nuevos algoritmos de tractografía que quizás proporcionen alguna ventaja sobre los anteriores, como es el ejemplo de la enfermedad de Alzheimer, donde la precisión diagnóstica varía en un mismo individuo, mediante la aplicación de nueve algoritmos de tractografía. ${ }^{19,20}$

Lo anteriormente expuesto pone de manifiesto dos limitaciones del estudio. La primera es que se contó con una muestra pequeña de sujetos. Esa muestra pequeña no permite hacer afirmaciones en cuanto a diferencias volumétricas por sexo, edad u otro tipo de características demográficas de la población. Sin embargo, resaltamos la dificultad de realizar tractografía en sujetos sanos, teniendo en cuenta las necesidades y limitaciones del sistema de salud que no permite grandes inversiones en población sana.

Además, las siembras manuales que se hicieron no garantizan que se incluya el $100 \%$ de las fibras de sustancia blanca que conforman el fascículo. Sin embargo, mediante las herramientas elipsoide y cuboide de FiberNavigator, se logró obtener el máximo de fibras y, lo más importante, mediante las ROI que se escogieron no se perdió el recorrido ni simetría del tracto.

Por otro lado, no contamos en el estudio con una herramienta que visualice las terminales corticales de cada fascículo estudiado, para que, de esa manera, se pueda realizar una correlación funcional y poder determinar una red cortico subcortical. Sin embargo, Revely y col., ${ }^{21}$ afirma que la compleja disposición de las fibras de materia blanca, implica en un desafío al intentar determinar sus conexiones corticales.

Dentro de lo que consideramos las ventajas del estudio mencionamos que, mediante la secuencia de pasos del procesado, se consiguió suprimir artefactos derivados de la adquisición y estandarizar las medidas, así como también se logró cegar a los investigadores, teniendo en cuenta que las imágenes ingresaban al estudio sin conocerse nombre, sexo, ni otras características demográficas del sujeto.

No se pretende que los hallazgos logrados sean un atlas de referencia para la visualización de tractos cerebrales, ya que de esos existen algunos, sino perfilar a la tractografía como una herramienta actual en el estudio de neuroimagen, para apoyo y diagnóstico precoz a la hora de investigar pacientes con alteraciones cognitivas. También se plantea la necesidad de crear una herramienta electrónica que permita la visualización de los fascículos cerebrales y de otras estructuras del cerebro de manera automática, ya que las siembras manuales implican inversión de tiempo y pueden estar sujetas al error humano.

\section{Conclusión}

Aunque esos resultados no sirven como valores de referencia para ser aplicados en pacientes con patología neurológica, por la gran variabilidad entre los diferentes equipos, hecho que limita la obtención de valores de referencia, brindamos información inexistente hasta el momento, con este software y la reproducción entre los distintos usuarios. Se plantea la necesidad de crear una herramienta electrónica automática y reproducible para el diagnóstico precoz y pronóstico de pacientes con alteración cognitiva.

Protección de personas y animales

Los autores declaran que para esta investigación no se han realizado experimentos en seres humanos ni en animales.

Confidencialidad de los datos

Los autores declaran que en este artículo no aparecen datos de pacientes.

Derecho a la privacidad y consentimiento informado. Los autores declaran que en este artículo no aparecen datos de pacientes. 


\section{Conflicto de intereses}

Los autores declaran no tener ningún conflicto de intereses.

\section{Bibliografía}

1 Caverzasi E, Papinutto N, Amirbekian B, Berger MS, Henry RG. Q-ball of inferior fronto-occipital fasciculus and beyond. PLoS One 2014;9 (06):e100274

2 Valeo T. News From The Society For Neuroscience Annual Meeting: Mapping the Connectome - the New Frontier in Neuroscience. Neurol Today 2014;14(05):34-36

3 Martino J, De Witt Hamer PC, Vergani F, et al. Cortex-sparing fiber dissection: an improved method for the study of white matter anatomy in the human brain. J Anat 2011;219(04):531-541

4 Urriola TMJ. Y TMJU, C RW. Más allá del tensor de difusión: Experiencia preliminar en Tractografía HARDI-CSD y SuperResolución en 1.5T. Rev Chil Radiol 2013;19(04):166-173

5 Zhu D, Zhang T, Jiang X, et al. Fusing DTI and fMRI data: a survey of methods and applications. Neuroimage 2014;102(Pt 1):184-191

6 Farshidfar Z, Faeghi F, Mohseni M, Seddighi A, Kharrazi HH, Abdolmohammadi J. Diffusion tensor tractography in the presurgical assessment of cerebral gliomas. Neuroradiol J 2014;27 (01):75-84

7 Zhu FP, Wu JS, Song YY, et al. Clinical application of motor pathway mapping using diffusion tensor imaging tractography and intraoperative direct subcortical stimulation in cerebral glioma surgery: a prospective cohort study. Neurosurgery 2012;71(06): 1170-1183, discussion 1183-1184

8 García-Pallero MA, Torres CV, Manzanares-Soler R, Cámara E, Sola RG. Papel de la imagen por tensor de difusion en el estudio prequirurgico de la epilepsia del lobulo temporal. Rev Neurol 2016;63(12):537-542

9 Larroza A, Moratal D, D’ocón Alcañiz V, Arana E; por la Alzheimer's Disease Neuroimaging Initiative. Tractografía del fascículo uncinado y el fascículo uncinado posterior en pacientes con deterioro cognitivo leve y enfermedad de Alzheimer. Neurologia 2014;29(01):11-20

10 Chamberland M, Bernier M, Fortin D, Whittingstall K, Descoteaux M. 3D interactive tractography-informed resting-state fMRI connectivity. Front Neurosci 2015;9:275
11 Wakana S, Caprihan A, Panzenboeck MM, et al. Reproducibility of quantitative tractography methods applied to cerebral white matter. Neuroimage 2007;36(03):630-644

12 Catani M, Thiebaut de Schotten M. A diffusion tensor imaging tractography atlas for virtual in vivo dissections. Cortex 2008;44 (08):1105-1132

13 Correia S, Lee SY, Voorn T, et al. Quantitative tractography metrics of white matter integrity in diffusion-tensor MRI. Neuroimage 2008;42(02):568-581

14 Bartrés-Faz D, Clemente IC, Junqué C. Cambios en la sustancia blanca y rendimiento cognitivo en el envejecimiento. Rev Neurol 2001;33(04):347-353

15 Michielse S, Coupland N, Camicioli R, et al. Selective effects of aging on brain white matter microstructure: a diffusion tensor imaging tractography study. Neuroimage 2010;52(04):1190-1201

16 Vassal F, Schneider F, Boutet C, Jean B, Sontheimer A, Lemaire JJ. Combined DTI tractography and functional MRI study of the language connectome in healthy volunteers: Extensive mapping of white matter fascicles and cortical activations. PLoS One 2016; 11(03):e0152614

17 Thiebaut de Schotten M, Ffytche DH, Bizzi A, et al. Atlasing location, asymmetry and inter-subject variability of white matter tracts in the human brain with MR diffusion tractography. Neuroimage 2011;54(01):49-59

18 den Braber A, van't Ent D, Stoffers D, Linkenkaer-Hansen K, Boomsma DI, de Geus EJC. Sex differences in gray and white matter structure in agematched unrelated males and females and opposite-sex siblings. Int J Psychol Res (Medellin) 2013; 6:7-21

19 Dell'Acqua F, Catani M. Structural human brain networks: hot topics in diffusion tractography. Curr Opin Neurol 2012;25(04): 375-383

20 Zhan L, Zhou J, Wang Y, et al; For The Alzheimer's Disease Neuroimaging Initiative. Comparison of nine tractography algorithms for detecting abnormal structural brain networks in Alzheimer's disease. Front Aging Neurosci 2015;7:48

21 Reveley C, Seth AK, Pierpaoli C, et al. Superficial white matter fiber systems impede detection of long-range cortical connections in diffusion MR tractography. Proc Natl Acad Sci U S A 2015;112(21): E2820-E2828 